\title{
Knockdown of IGF2R suppresses proliferation and induces apoptosis in hemangioma cells in vitro and in vivo
}

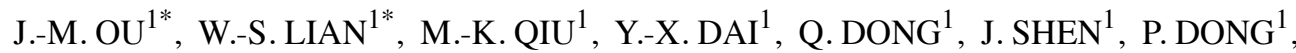 \\ X.-F. WANG ${ }^{1}$, Y.-B. LIU ${ }^{1}$, Z.-W. QUAN ${ }^{1}$ and Z.-W. FEI ${ }^{2}$ \\ ${ }^{1}$ Department of General Surgery, Xinhua Hospital Affiliated to Shanghai Jiaotong University School of Medicine, \\ Shanghai 200092; ${ }^{2}$ Department of General Surgery, Xinhua Hospital (Chong Ming) Affiliated to \\ Shanghai Jiaotong University School of Medicine, Shanghai 202150, P.R. China
}

Received March 27, 2014; Accepted May 26, 2014

DOI: 10.3892/ijo.2014.2512

\begin{abstract}
Insulin-like growth factor-II (IGF-II)/IGF2R signaling plays a pivotal role in cell growth, migration and differentiation in many malignancies. An individual with high IGF-II expression levels has a high risk of developing cancer, but IGF2R is often considered to be a tumor suppressor. To date, little has been reported about the role of IGF-II/IGF2R signaling in hemangiomas (HAs). Thus, uncovering the mechanisms of IGF-II/IGF2R signaling is very important to understanding the development of HAs. In the present study, the expression of IGF-II and IGF2R was investigated in 27 cases of HAs of different phases by immunohistochemistry. Through lentivirus-mediated IGF2R siRNA (Lv-siIGF2R) in HA-derived endothelial cells (HDECs), we observed the effects of IGF2R knockdown on the biological behavior of HA cells. We found that the expression of IGF-II and IGF2R was significantly increased in proliferating phase HAs, but decreased in involuting phase HAs. Furthermore, knockdown of IGF2R in vitro significantly diminished the proliferative activity and induced apoptosis and cycle arrest with decreased expression of PCNA, Ki-67, Bcl-2, Cyclin D1 and E and increased the expression of Bax in the proliferative phase HAs (HDEC and CRL-2586 EOMA cells). In addition, the tumor volumes in a subcutaneous HDEC nude mouse model treated with Lv-siIGF2R were significantly smaller than those of the control group. Taken together, our findings indicate that the expression of IGF-II and IGF2R is increased in proliferating
\end{abstract}

Correspondence to: Professor Zhe-Wei Fei, Department of General Surgery, Chongming Branching Hospital, Xinhua Hospital Affiliated to Shanghai Jiaotong University School of Medicine, Shanghai Chongming County, 25 Nan men gang Road, Shanghai 202150, P.R. China

E-mail: zheweifei@xinhuamed.com.cn

*Contributed equally

Key words: insulin-like growth factor-II, IGF2R, hemangioma, proliferation, apoptosis phase HAs, and knockdown of IGF2R suppresses proliferation and induces apoptosis in HA cells in vitro and in vivo, suggesting that IGF2R may represent a novel therapeutic target for the treatment of human HAs.

\section{Introduction}

Hemangiomas (HAs) are one of the most common benign tumors, but the prevalence and the pathogenesis of HAs are not well understood. HAs are characterized by three phases: proliferating, involuting and involuted phases, and is defined by a period of rapid proliferation of blood vessels in the first year of life, followed by gradual regression of the vascular component with eventual replacement by fibro-fatty tissue (1).

Insulin-like growth factor-II (IGF-II) plays an important role in tumor development and progression through activation of the receptor IGF2R. IGF-II is expressed in cholangiocarcinoma (CC) cell lines, and inhibition of IGF-II by the growth factor inhibitor influences the oncogene K-RAS genotype in CC (2). Integrative genomic analysis shows IGF-II/ IGF2R signaling is activated in the proliferation subclass of hepatocellular carcinoma (HCC), and effective blockage of IGF1R signaling also provides the rationale for testing $\mathrm{HCC}$ therapy in clinical trials (3). Also, the polymorphic variants of IGF-axis genes act alone or jointly with other risk factors to affect susceptibility to pancreatic cancer, and are used to identify population subgroups that benefit from IGF2R-targeted agents $(4,5)$.

Thus, abnormalities in IGF-II/IGF2R signaling are associated with the development of a wide variety of tumors (6). Increased IGF-II expression stimulates proliferation and results in a rapid conversion to malignancy during tumor formation in primary mouse embryonic fibroblasts (7). The 3' UTR IGF2R-A2/B2 variant is associated with increased tumor growth and advanced stages in non-small cell lung cancer (8), and genetic variation of IGF2R may advance distant metastasis, and influence the risk of developing some cancers (9-11). Therefore, although there is insufficient evidence supporting the associations between IGF1R single-nucleotide polymorphisms (rs2272037) and brain tumor risk (12), interaction of IGF-II with IGF2R increases the risk of malignant transformation and enhances the aggressive breast cancer phenotype, 
suggesting that IGF2R may serve as a potential therapeutic target for HAs (13).

However, some studies have shown that mannose 6-phosphate/insulin-like growth factor II receptor (M6P/IGF2R) gene can function as the tumor suppressor in cancer. M6P/IGF2R is found inactivated in prostate cancer and the mutation of this gene is an early event in the development of prostate cancer (14). M6P/IGF2R also functions as a growth suppressor, but the loss or mutation of this gene contributes to development and progression of some cancers $(15,16)$ and is involved in HBV-associated hepatocarcinogenesis (17). Moreover, M6P/ IGF2R controls cell adhesion and invasion by regulating $\alpha \mathrm{v}$ integrin expression and accelerating uPAR cleavage (18), and restricts the metastatic propensity of squamous cell carcinomas (19). These studies thereby support that M6P/IGF2R may act as the tumor suppressor in carcinogenesis.

In terms of the multi-functionality of IGF2R in cancer, exploration of the expression and function of IGF-II/IGF2R signaling in HAs is indispensable. In the present study, tissues from 27 cases of HA tissues of different phases were collected, and the expression of IGF-II and IGF2R was examined. Using lentivirus-mediated IGF2R knockdown in HA-derived endothelial cells (HDEC), we investigated the effects of IGF2R knockdown on the biological behavior of human HA cells. We found that the expression of IGF-II and IGF2R was increased in proliferating phase HAs, and knockdown of IGF2R repressed proliferation and induced apoptosis in HA cells in vitro and in vivo, suggesting that IGF2R might represent a novel therapeutic target for the treatment of human HAs.

\section{Materials and methods}

Materials. The primary HDEC and CRL-2586 EOMA cells used in these experiments were from the Institute of Biochemistry and Cell Biology (Shanghai, China). The lentivirus vector Lv-siIGF2R, negative control vector (scramble-siRNA) and virion-packaging elements were from Genechem (Shanghai, China). The IGF2R primer was synthesized by ABI (Framingham, MA, USA) and all antibodies were from Santa Cruz Biotechnology (Dallas, TX, USA).

Drugs and reagents. Dulbecco's modified Eagle's medium (DMEM) and fetal bovine serum (FBS) were from Thermo Fisher Scientific Inc. (Waltham, MA, USA); TRIzol reagent and Lipofectamine 2000 were from Invitrogen (Carlsbad, CA, USA); M-MLV reverse transcriptase was from Promega (Madison, WI, USA); SYBR Green Master Mixture was from Takara (Otsu, Japan). ECL-Plus/kit was from GE Healthcare (Piscataway, NJ, USA). Cell cycle analysis kit and apoptosis kit [propidium iodide (PI), RNase A, Annexin V-FITC] were from KeyGen Biology (Nanjing, China).

Tissue samples. Twenty-seven freshly resected human HAs and 18 cases of adjacent normal skin tissue samples were collected at the Department of General Surgery, and were classified according to Mulliken criteria. Tissues and clinical information were obtained as part of an approved study at Shanghai Jiao Tong University School of Medicine. There were 15 cases of proliferating phase HAs and 12 cases of involuting phase HAs. A portion of each tissue sample was fixed with
$10 \%$ formalin for histopathological and IHC examination. All HA tissues were diagnosed by two independent pathologists.

IHC staining. IHC staining for IGF-II (rabbit polyclonal, ab9572, Abcam, MA, USA), IGF2R (rabbit polyclonal A0545, Sigma, USA) and PCNA (mouse monoclonal PC10, Sigma) using polyclonal and monoclonal antibodies was performed. Unstained sections were deparaffinized and incubated overnight at $4^{\circ} \mathrm{C}$ with primary antibodies against the proteins, then with biotinylated secondary antibody (1:200) at room temperature for $1 \mathrm{~h}$, followed by incubation with $\mathrm{ABC}$ peroxidase and 3,3' diaminobenzidine (DAB; $30 \mathrm{mg}$ dissolved in $100 \mathrm{ml}$ Tris-buffer containing $0.03 \% \mathrm{H}_{2} \mathrm{O}_{2}$ ). Sections were counterstained with hematoxylin. Digital images were acquired and the integrated optical density (IOD) and positive expression rate (cell positive area/cell total area) of semi-quantitative analysis was performed.

Cell culture and transfection. The proliferating phase HDEC and CRL-2586 EOMA cells were cultured in DMEM medium supplemented with $10 \%$ heat-inactivated FBS, $100 \mathrm{U} / \mathrm{ml}$ of penicillin and $100 \mu \mathrm{g} / \mathrm{ml}$ of streptomycin. They were all placed in a humidified atmosphere containing $5 \% \mathrm{CO}_{2}$ at $37^{\circ} \mathrm{C}$. Lv-siIGF2R and negative control viruses were transfected into proliferating phase HDEC and CRL-2586 EOMA cells. Cells were subcultured at a 1:5 dilution in $300 \mu \mathrm{g} / \mathrm{ml}$ G418-containing medium. On the day of transduction, HA cells were replated at $5 \times 10^{4}$ cells/well in 24 -well plates containing serum-free growth medium with polybrene $(5 \mathrm{mg} / \mathrm{ml})$. When the cells reached $50 \%$ confluence, they were transfected with recombinant experimental virus or control virus at the optimal MOI (multiplicity of infection) of 50, and cultured at $37^{\circ} \mathrm{C}$ and $5 \% \mathrm{CO}_{2}$ for $4 \mathrm{~h}$. Then supernatant was discarded and serum containing growth medium was added. At 4 days of post-transduction, transduction efficiency was measured by the frequency of green fluorescent protein (GFP)-positive cells. Positive stable transfectants were selected and expanded for further study. The clones transfected with the Lv-siIGF2R vectors were named as the Lv-siIGF2R group, and the clones transfected with the negative control vectors was named as the NC group.

Quantitative real-time PCR. To quantitatively determine the mRNA expression level of IGFII and IGF2R in proliferating phase HA tissues and cells, Real-time PCR was used. Total RNA of each clone was extracted with TRIzol according to the manufacturer's protocol. Reverse-transcription was carried out using M-MLV and cDNA amplification was carried out using SYBR Green Master Mix kit according to the manufacturer's protocol. The IGF2R gene was amplified using specific oligonucleotide primer and human glyceraldehyde-3-phosphate dehydrogenase (GAPDH) gene was used as an endogenous control. The PCR primer sequences were listed as follows: IGFII, 5'-AGAGAGGCCAAACGTCATCGT-3' and 5'-TCACC CCACCTTGCAGAATTA-3'; IGF2R, 5'-GAAAACACAAG AATGAAGGTCAA-3' and 5'-CCACCAGCTCTCCTCCAC ATA-3'; GAPDH, 5'-CAACGAATTTGGCTACAGCA-3' and 5'-AGGGGTCTACATGGCAACTG-3'. Data were analyzed using the comparative $\mathrm{Ct}$ method $\left(2^{-\Delta \Delta \mathrm{Ct}}\right)$. Three separate experiments were performed for each clone. 
Western blot assay. The proliferating phase HDEC and CRL-2586 EOMA cells were harvested and extracted using lysis buffer (Tris-HCl, SDS, mercaptoethanol, glycerol). Cell extracts were boiled for $5 \mathrm{~min}$ in loading buffer and then an equal amount of cell extracts from each sample was separated on $15 \%$ SDS-PAGE gels. Separated protein bands were transferred onto poly vinylidene fluoride (PVDF) membranes and the membranes were blocked in 5\% skim-milk powder dissolved in PBST. The primary antibodies against IGF-II (rabbit polyclonal ab9572, Abcam), IGF2R (rabbit polyclonal A0545, Sigma), PCNA (mouse monoclonal PC10, Sigma), Ki-67 (rabbit polyclonal ab888, Abcam), Bcl-2 (rabbit monoclonal SP66, Sigma), Bax (rabbit monoclonal ab32503, Abcam) and Cyclin D1 (rabbit monoclonal sc-718, Santa Cruz, USA) were diluted according to the manufacturer's instructions of antibodies and incubated overnight at $4{ }^{\circ} \mathrm{C}$. Then, horseradish peroxidase-linked secondary antibodies were added at a dilution ratio of 1:1,000, and incubated at room temperature for $2 \mathrm{~h}$. The membranes were washed three times with PBS for three times and the immunoreactive bands were visualized using ECL-Plus/kit according to the manufacturer's instructions. The relative protein level in different cell lines was normalized to the endogenous level of $\beta$-actin protein. Three separate experiments were performed for each clone.

Cell proliferation assay. Cell proliferation was analyzed with the MTT assay. Briefly, cells infected with Lv-siIGF2R were incubated in 96-well-plates ( 8 wells each group) at a density of $1 \times 10^{5}$ cells per well with DEME medium supplemented with $10 \%$ FBS. Cells were treated with $20 \mu \mathrm{l}$ MTT dye at $0,24,48$, and $72 \mathrm{~h}$ and then incubated with $150 \mu \mathrm{l}$ of DMSO for $5 \mathrm{~min}$. The color reaction was measured at $570 \mathrm{~nm}$ with enzyme immunoassay analyzer (Bio-Rad, USA). The proliferation activity was calculated for each clone.

Cell apoptosis analysis. To detect cell apoptosis, the proliferating phase HDEC and CRL-2586 EOMA cells were trypsinized, washed with cold PBS and resuspended in binding buffer according to the instruction of the apoptosis kit. FITC-Annexin V and PI were added to the fixed cells for $20 \mathrm{~min}$ in darkness at room temperature. Then, Annexin V binding buffer was added to the mixture before the fluorescence was measured on FACsort flow cytometer (Becton-Dickinson, Mountain View, CA, USA). The cell apoptosis was analyzed using CellQuest software (Becton-Dickinson). Cell apoptotic rate $=$ the number of cell apoptosis/the number of apoptotic cells and the normal cells. Three separate experiments were performed for each clone.

Cell cycle analysis. To detect cell cycle variation, the proliferating phase HDEC and CRL-2586 EOMA cells were trypsinized, washed with PBS and fixed with $80 \%$ cold ethanol overnight at $-20^{\circ} \mathrm{C}$. After another wash with PBS, the fixed cells were stained with PI in the presence of RNase A for $30 \mathrm{~min}$ at room temperature in darkness. Each sample was filtered through a 50- $\mu \mathrm{m}$ nylon filter to obtain single-cell suspension. The samples were then analyzed on FACsort flow cytometer (Becton-Dickinson). ModFit3.0 software (Verity Software House, Topsham, ME, USA) was used for cell cycle analysis. Three separate experiments were performed for each clone.

Subcutaneous tumor model and gene therapy. Six-week-old female immune-deficient nude mice (BALB/c-nu) were bred at the laboratory animal facility (Institute of Chinese Academy of Sciences, Shanghai, China), and were housed individually in microisolator ventilated cages with free access to water and food. All experimental procedures were performed according to the regulations and internal biosafety and bioethics guidelines of Shanghai Jiaotong University and the Shanghai Municipal Science and Technology Commission.

Four mice were injected subcutaneously with $1 \times 10^{7}$ HDEC cells in $50 \mu \mathrm{l}$ of PBS pre-mixed with an equal volume of Matrigel matrix (Becton-Dickinson). Mice were monitored daily, and three out of four mice developed a subcutaneous tumor. When the tumor size reached $\sim 5 \mathrm{~mm}$ in length, it was surgically removed, cut into $1-2 \mathrm{~mm}^{3}$ pieces, and re-seeded individually into other mice on the right flanks. When tumor size reached $\sim 5 \mathrm{~mm}$ in length, the mice were randomly assigned as PBS group, negative control (NC) group and Lv-siIGF2Rtreated group. In treatment group, $15 \mu \mathrm{l}$ of Lv-siIGF2R was injected into subcutaneous tumors using a multi-site injection format (at least three sites). Mice in the PBS (HDEC) group and the NC group received $15 \mu \mathrm{l}$ of PBS with or without negative control lentivirus, respectively. Injections were repeated every other day after initial treatment. The tumor volume was measured every three days with a caliper, using the formula volume $=(\text { length } \mathrm{x} \text { width })^{2} / 2$.

Statistical analysis. SPSS 20.0 was used for the statistical analysis. One-way analysis of variance (ANOVA) was used to analyze the differences between groups. The LSD method of multiple comparisons was used when the probability for ANOVA was statistically significant. Statistical significance was $\mathrm{P}<0.05$. In the figures, the error bars depict standard deviation.

\section{Results}

The expression of IGF-II, IGF2R and PCNA in human $H A s$. The protein expression of IGF-II, IGF2R and PCNA was evaluated using IHC analysis. As shown in Fig. 1A, the positive expression of IGF-II, IGF2R and PCNA was found increased in proliferating phase HAs, but decreased in involuting phase HAs. From the cell localization, the positive staining of IGF-II was mainly in the cytoplasm, that of IGF2R was in the membrane and cytoplasm, but PCNA was in the cellular nucleus in HA and normal tissue cells. The average IOD and positive rates of IGF-II, IGF2R and PCNA were significantly higher in proliferating phase HAs than those in involuting phase HAs or in normal tissues (Table I, each $\mathrm{P}<0.01$ ). Spearman correlation analysis showed the positive correlation between the IGF-II, IGF2R and PCNA expression in HA tissues of different phases $(r=0.625, \mathrm{P}=0.015)$. Then, we examined the mRNA and protein expression levels of IGF-II and IGF2R in HA tissues by real-time PCR (Fig. 1B and C) and western blot assays (Fig. 1D and E), and further found that the expression of IGF-II and IGF2R was increased in proliferating phase HAs, but decreased in involuting phase HA. 


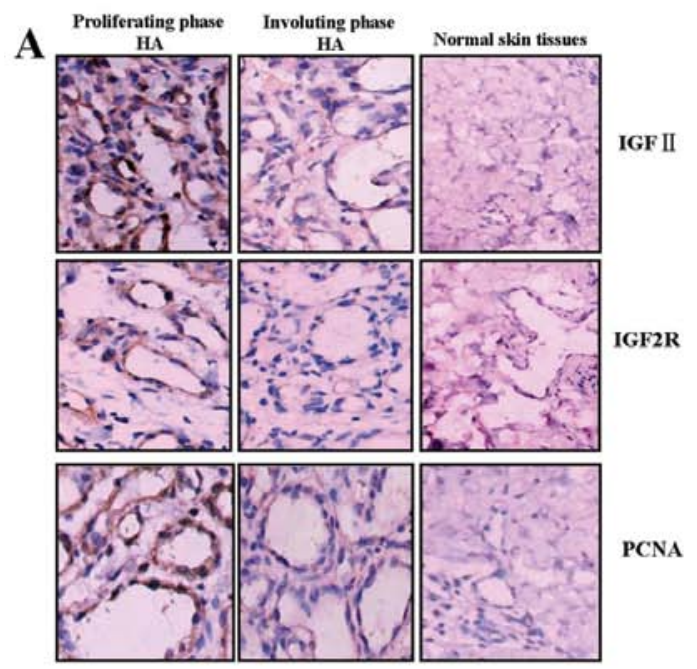

B

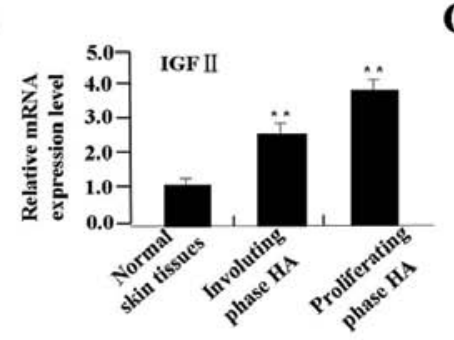

D

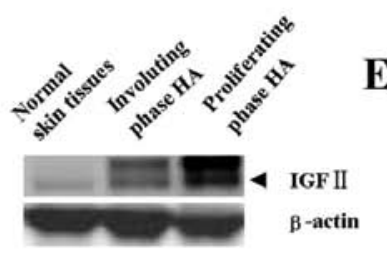

C

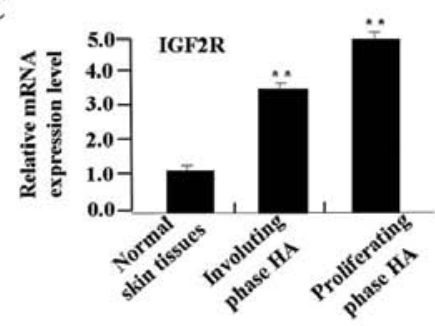

$\mathbf{E}$

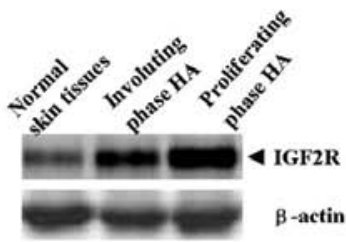

Figure 1. The expression of IGF-II, IGF2R and PCNA in different phase HAs. (A) The positive expression rates of IGF-II, IGF2R and PCNA, examined by IHC staining, were increased in proliferating HA tissues, but decreased in involuting phase HAs. The mRNA and protein expression levels of IGF-II and IGF2R were detected by real-time PCR (B and C) and western blot assays (D and E) in HA tissues, indicating that the expression of IGF-II and IGF2R was increased in proliferating phase HAs, but decreased in involuting phase HAs.

Table I. Expression of IGFII, IGF2R and PCNA in different phase HAs.

\begin{tabular}{|c|c|c|c|c|c|c|c|}
\hline \multirow[b]{2}{*}{ Group } & \multirow[b]{2}{*}{ Case } & \multicolumn{2}{|c|}{ IGFII } & \multicolumn{2}{|c|}{ IGF2R } & \multicolumn{2}{|c|}{ PCNA } \\
\hline & & IOD & Positive rate & IOD & Positive rate & IOD & Positive rate \\
\hline Normal skin tissues & 18 & $2.53 \pm 0.67$ & $0.04 \pm 0.01$ & $3.65 \pm 0.71$ & $0.02 \pm 0.01$ & $2.15 \pm 0.82$ & $0.02 \pm 0.01$ \\
\hline Involuting phase HAs & 12 & $7.29 \pm 1.17^{\mathrm{a}}$ & $0.12 \pm 0.03^{\mathrm{a}}$ & $10.78 \pm 2.05^{\mathrm{a}}$ & $0.09 \pm 0.03^{\mathrm{a}}$ & $8.16 \pm 2.19^{\mathrm{a}}$ & $0.10 \pm 0.03^{\mathrm{a}}$ \\
\hline Proliferating phase HAs & 15 & $13.05 \pm 1.21^{\mathrm{ab}}$ & $0.18 \pm 0.04^{\mathrm{ab}}$ & $12.98 \pm 1.45^{\mathrm{ab}}$ & $0.13 \pm 0.03^{\mathrm{ab}}$ & $13.01 \pm 2.12^{\mathrm{ab}}$ & $0.15 \pm 0.04^{\mathrm{ab}}$ \\
\hline
\end{tabular}

HAs, hemangiomas. ${ }^{\mathrm{a}} \mathrm{P}<0.01$ vs. normal skin tissues; ${ }^{\mathrm{b}} \mathrm{P}<0.01$ vs. involuting phase HAs.

The siRNA knockdown efficiency for IGF2R expression. To examine the Lv-siIGF2R knockdown efficiency for IGF2R expression in proliferative phase HDEC and CRL-2586 EOMA cells, we investigated the expression levels of IGF2R in Lv-siIGF2R-transfected HA cells using real-time PCR and western blot assays. As shown in Fig. 2, the knockdown efficiency for IGF2R expression was found to be significantly increased in Lv-siIGF2R-transfected HDEC (Fig. 2A and C) and CRL-2586 EOMA cells (Fig. 2B and D), respectively reaching $>80$ and $70 \%$ (each ${ }^{* *} \mathrm{P}<0.01$ ).

Effect of Lv-siIGF2R on cell proliferation. To investigate the effect of Lv-siIGF2R on cell proliferation in HA cells (HDEC and CRL-2586 EOMA), we examined cell proliferative activities using MTT assay. We found that Lv-siIGF2R could significantly diminish the proliferative activity of HA cells in a time-dependent manner compared with the NC group (Fig. 3A and B). In addition, to determine whether IGF2R knockdown suppressed the endogenous expression of Ki-67 and PCNA, we examined the protein expression of Ki-67 and PCNA by western blot assay. The amount of Ki-67 and PCNA proteins was significantly decreased in the Lv-siIGF2R group compared with the NC group (each ${ }^{* *} \mathrm{P}<0.01$ ) (Fig. $3 \mathrm{C}$ and D), suggesting that knockdown of IGF2R might inhibit HA cell proliferation through downregulation of Ki-67 and PCNA expression.

Effect of Lv-siIGF2R on cell apoptosis. To determine whether IGF2R knockdown affected the apoptosis in HA cells (HDEC and CRL-2586 EOMA), flow cytometric analysis was performed. Cell apoptotic rates were markedly increased in Lv-siIGF2R group compared with the NC group $\left(\right.$ each $\left.^{* *} \mathrm{P}<0.01\right)$ (Fig. 4A and B). To determine whether IGF2R knockdown influences the expression of Bax and Bcl-2, we examined the expression of Bax and Bcl-2 in HA cells by western blot assay. It was found that the amount of Bax was markedly increased, while that of Bcl-2 protein was decreased in the Lv-siIGF2R group compared with the NC group (each ${ }^{* *} \mathrm{P}<0.01$ ) (Fig. 4C and $\mathrm{D}$ ), suggesting that knockdown of IGF2R might induce HA cell apoptosis through regulation of the expression of Bax and Bcl-2.

Effect of Lv-siIGF2R on cell cycle distribution. To determine whether IGF2R knockdown influenced cycle distribution in HA cells (HDEC and CRL-2586 EOMA), flow cytometry analysis was performed. We showed that the $G_{0} / G_{1}$ phase 


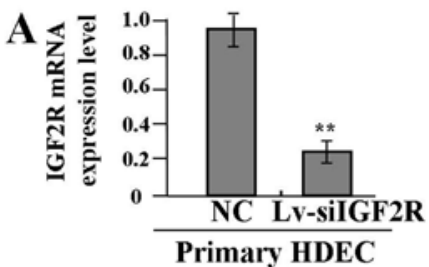

C

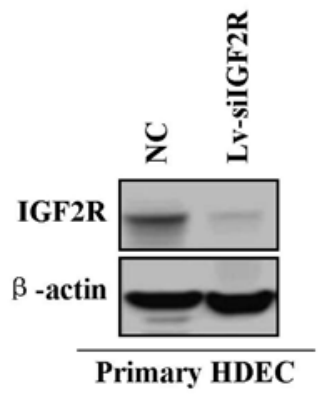

D

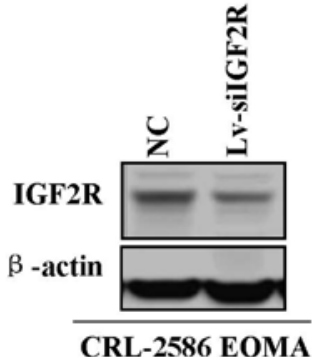

B
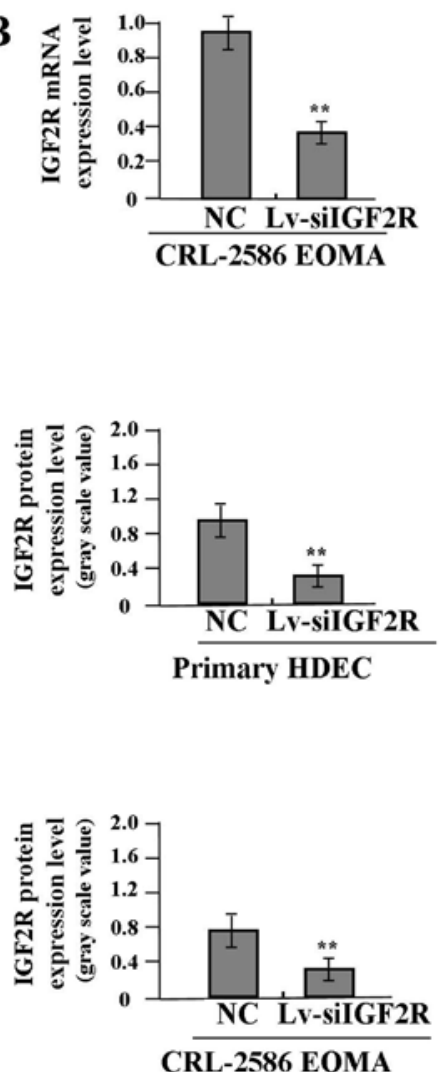

Figure 2. The siRNA knockdown efficiency for IGF2R expression. Real-time PCR and western blot assays were performed to assess the siRNA knockdown efficiency, which was found to be significantly increased in Lv-siIGF2R-transfected HDEC (A and C) and CRL-2586 EOMA cells (B and D), respectively reaching $>80$ and $70 \%\left(\right.$ each $\left.^{* *} \mathrm{P}<0.01\right)$.

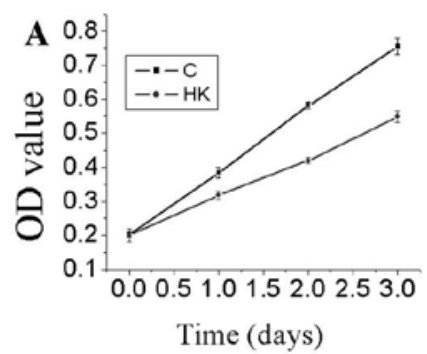

Primary HDEC

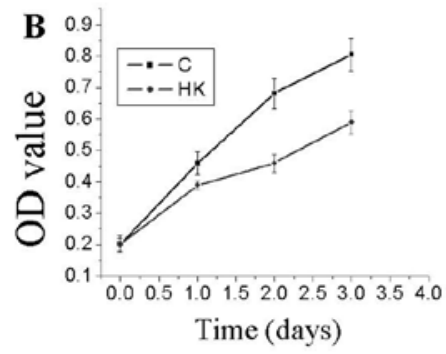

CRL-2586 EOMA

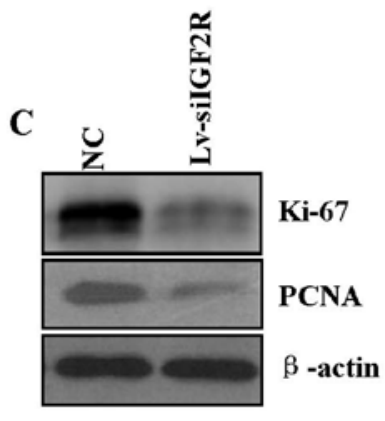

Primary HDEC
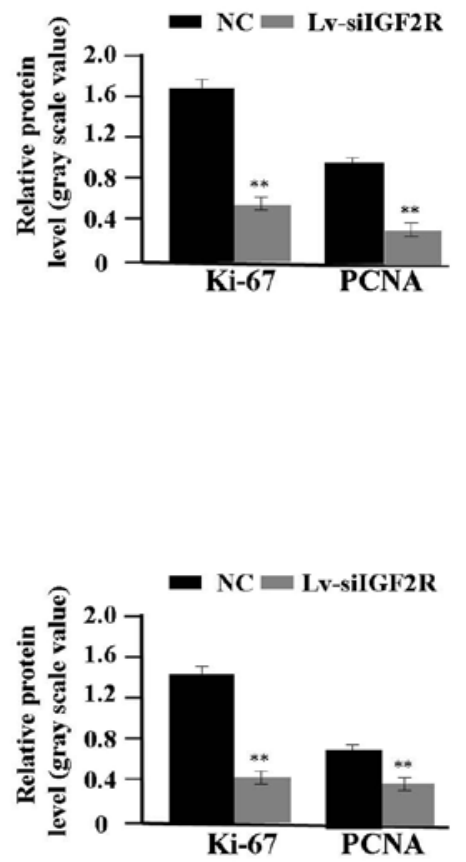

CRL-2586 EOMA

Ki-67

PCNA

$\beta$-actin

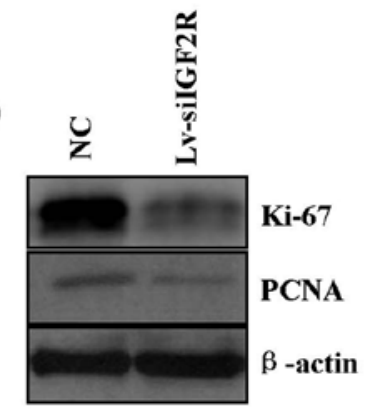

Figure 3. Effect of Lv-siIGF2R on cell proliferation. (A and B) MTT assay was performed to examine cell proliferative activities. Lv-siIGF2R significantly diminished proliferative activities of HA cells in a time-dependent manner compared with the NC group, indicating that knockdown of IGF2R might inhibit HA cell proliferation. (C and D) Western blotting was carried out to assess protein expression levels of Ki-67 and PCNA protein. The amount of Ki-67 and PCNA protein was significantly decreased in Lv-siIGF2R group compared with the NC group (each $\left.{ }^{* *} \mathrm{P}<0.01\right)$. 

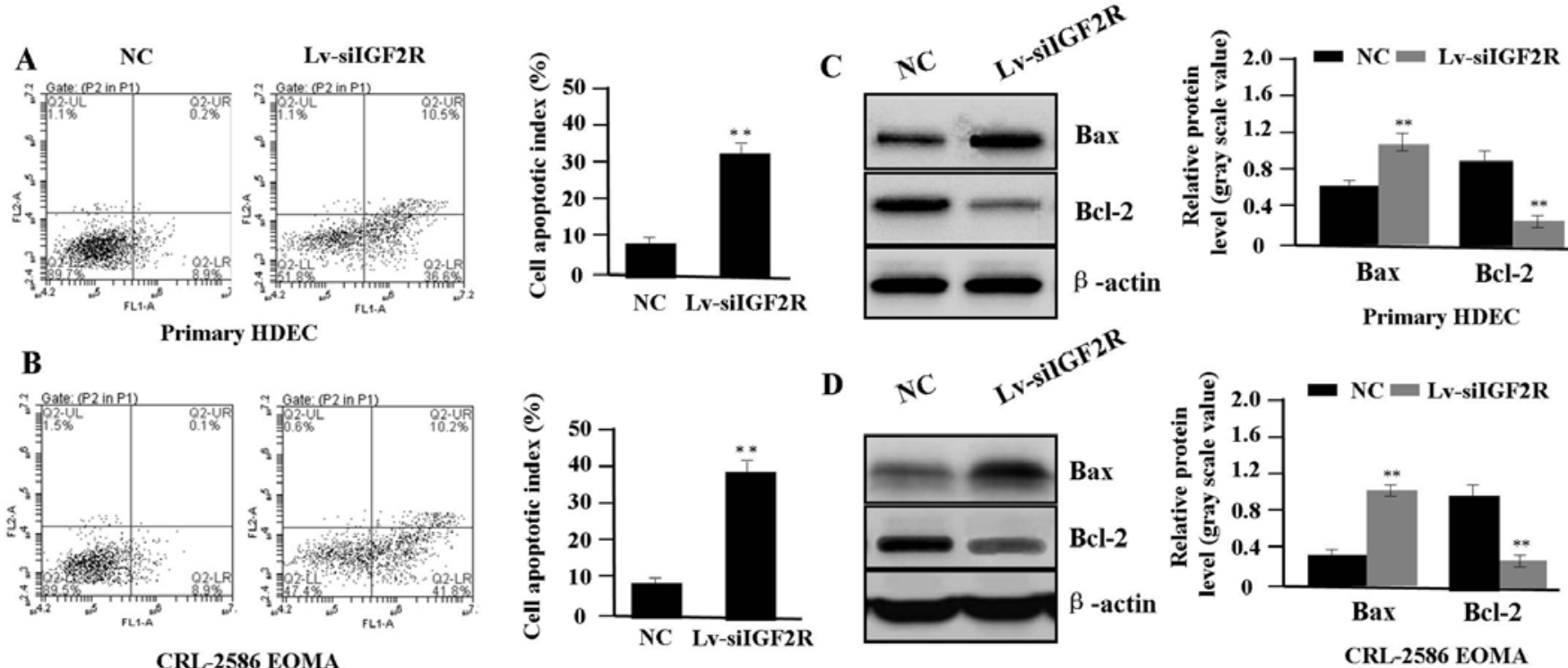

Figure 4. Effect of Lv-siIGF2R on cell apoptosis. (A and B) Flow cytometric analysis was performed to detect cell apoptosis. Cell apoptotic rates were significantly increased in Lv-siIGF2R group compared with the NC group in HA cells (each ${ }^{* *} \mathrm{P}<0.01$ ), indicating that knockdown of IGF2R induced HA cell apoptosis. (C and D) Western blotting was carried out to assess protein expression levels of Bax and Bcl-2 protein in HA cells. Expression of Bax was increased, but that of Bcl-2 was decreased in Lv-siIGF2R group compared with the $\mathrm{NC}$ group (each $\left.{ }^{* *} \mathrm{P}<0.01\right)$.
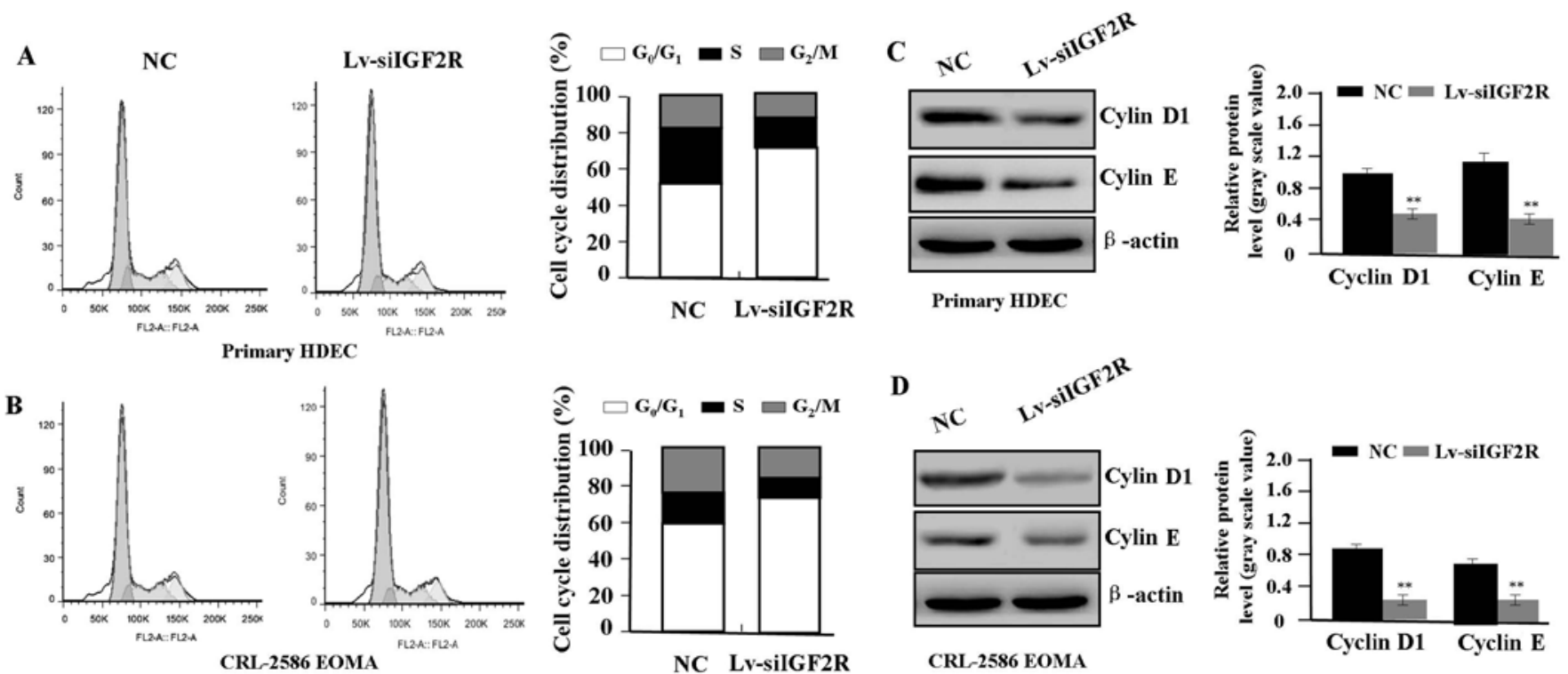

Figure 5. Effect of Lv-siIGF2R on cell cycle distribution. (A and B) Flow cytometric analysis was performed to determine cell cycle distribution, which showed that $\mathrm{G}_{0} / \mathrm{G}_{1}$ phase fraction was increased, but $S$ and $\mathrm{G}_{2} / \mathrm{M}$ phase fractions were decreased in the Lv-siIGF2R group compared with the $\mathrm{NC}$ group, indicating that knockdown of IGF2R induced cell cycles arrest in $\mathrm{G}_{0} / \mathrm{G}_{1}$ phase in HA cells. (C and D) Western blotting was used to assess protein expression levels of Bax and Bcl-2 protein in HA cells. Expression of Cyclin D1 and E was downregulated in Lv-siIGF2R group compared with the $\mathrm{NC}$ group $\left(\mathrm{each}^{* *} \mathrm{P}<0.01\right)$.

fraction was increased, while the $S$ and $\mathrm{G}_{2} / \mathrm{M}$ phase fractions were decreased, and more HA cells were arrested in the $\mathrm{G}_{0} / \mathrm{G}_{1}$ phase in the Lv-siIGF2R group compared with the NC group (Fig. 5A and B). In order to determine whether IGF2R knockdown suppressed the expression of Cyclin D1 and E, we examined the expression of Cyclin D1 and $\mathrm{E}$ by western blot assay. The amount of Cyclin D1 and E protein was markedly decreased in the Lv-siIGF2R group compared with the NC group (each ${ }^{* *} \mathrm{P}<0.01$ ) (Fig. 5C and $\mathrm{D}$ ), suggesting that knock- down of IGF2R might induce cycle arrest in HA cells through downregulation of Cyclin D1 and E expression.

Effect of Lv-siIGF2R on signaling transduction of PI3K/AKT. Many studies have demonstrated that the PI3K/AKT pathway is involved in regulating HA formation $(20,21)$. Whether Lv-siIGF2R affects PI3K/AKT signaling transduction of $\mathrm{PI} 3 \mathrm{~K} / \mathrm{AKT}$ needed to be explored. Western blot assays were performed to assess the expression of P-PI3K, P-AKT, PI3K 


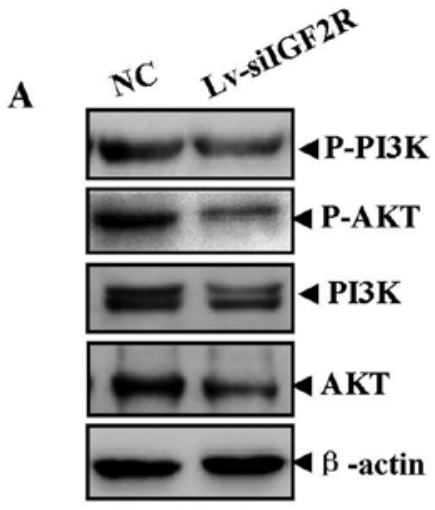

Primary HDEC

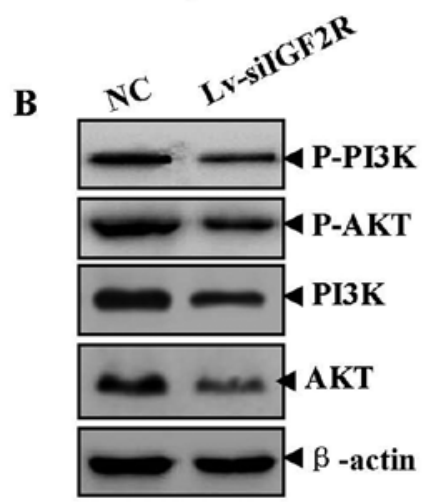

CRL-2586 EOMA
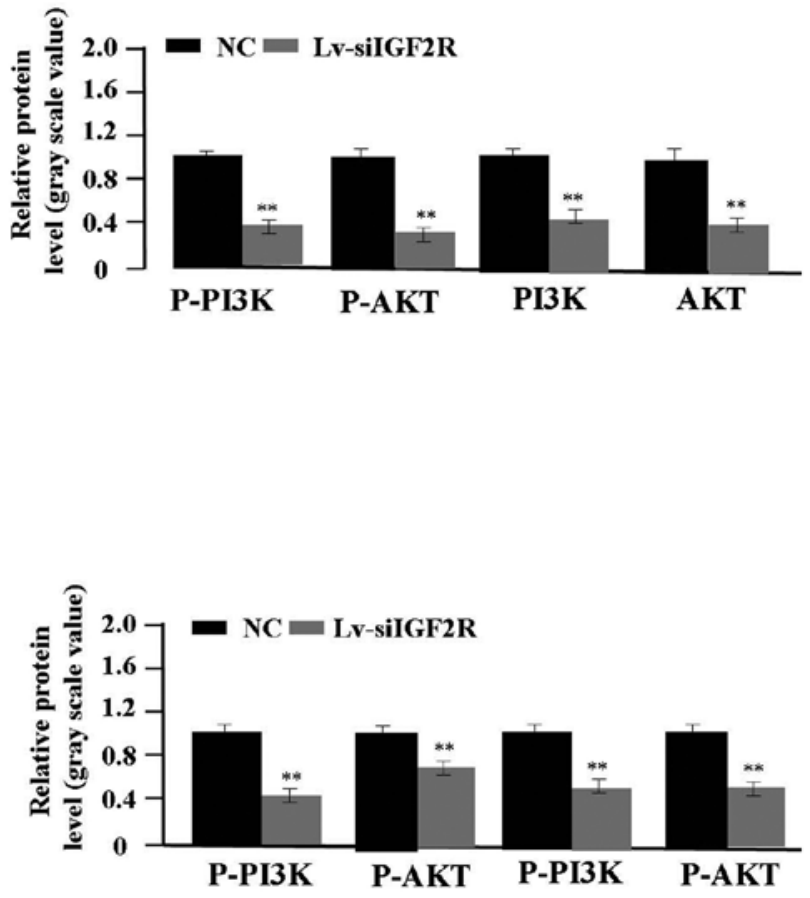

Figure 6. Effect of Lv-siIGF2R on signaling transduction of PI3K/AKT. (A and B) Western blotting was performed to assess protein expression levels of $\mathrm{P}-\mathrm{PI} 3 \mathrm{~K}$ and P-AKT in HA cells. Expression of p-PI3K and p-AKT protein was significantly downregulated in Lv-siIGF2R group compared with the NC group in HA cells $\left(\right.$ each $\left.{ }^{* *} \mathrm{P}<0.01\right)$.

A

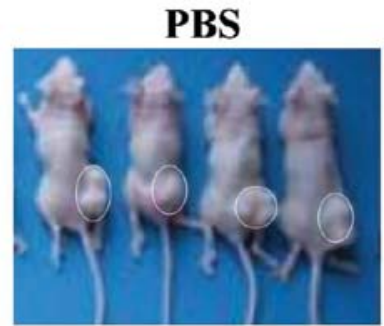

B

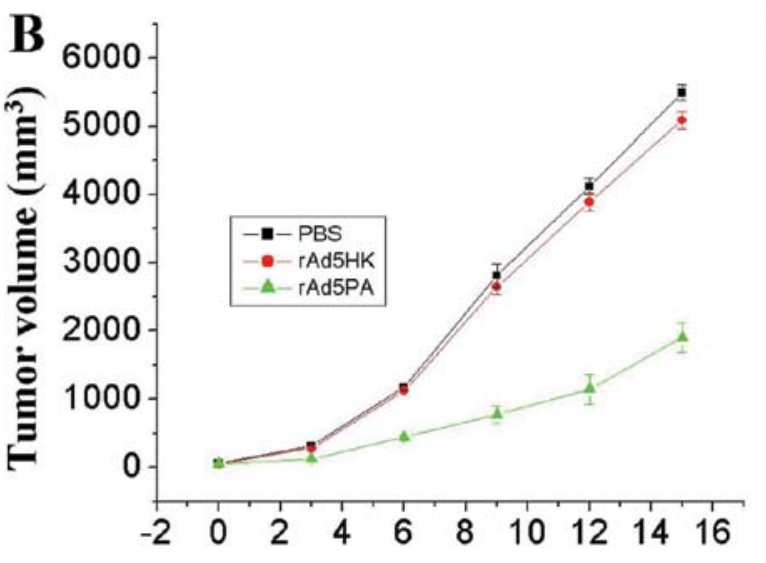

NC

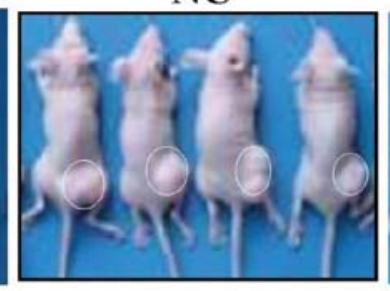

\section{Lv-siIGF2R}

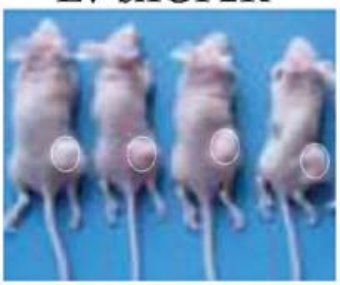

C

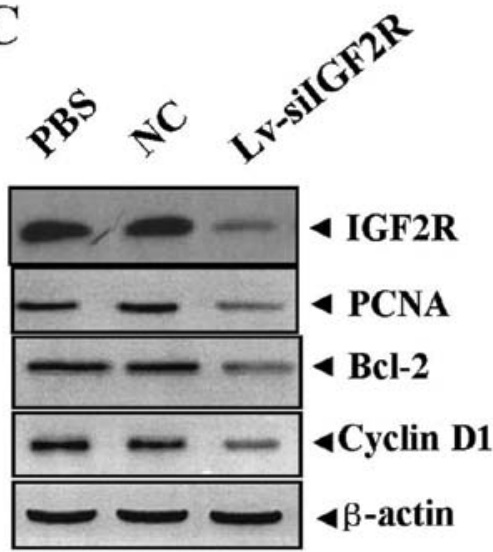

Time (days)

Figure 7. Antitumor effect of Lv-siIGF2R on HDEC xenograft model. (A) Over the observation period, tumor volumes were much smaller in the Lv-siIGF2R group than those in PBS and NC groups. (B) Tumor growth curve showed that the tumors in the Lv-siIGF2R group grew slowly compared with those in PBS and NC groups. (C) The tumor samples were removed for western blot analysis of IGF2R, PCNA, Bcl-2 and Cyclin D1 expression, indicating that their protein expression levels were significantly decreased in Lv-siIGF2R group compared with those in PBS and NC groups. 
and AKT in Lv-siIGF2R-transfected HA cells. We found that the activity of P-PI3K, P-AKT, PI3K and AKT proteins was reduced in the Lv-siIGF2R group compared with the NC group (each ${ }^{* *} \mathrm{P}<0.01$ ) (Fig. 6), suggesting that knockdown of IGF2R might block the PI3K/AKT signaling transduction in HA cells.

Antitumor effect of Lv-siIGF2R on the HDEC xenograft model. Our in vitro experiments demonstrated that knockdown of IGF2R could efficiently inhibit proliferation and induce apoptosis in HA cells. Therefore, we further investigated the antitumor effect of Lv-siIGF2R in vivo using the HDEC xenograft model and lentivirus-mediated gene therapy. The mean volume of tumors in all experimental mice before treatment was $78.30 \pm 8.20 \mathrm{~mm}^{3}$. During the first 3 days of recovery, the tumors in each group grew slowly, with no obvious difference in tumor size. However, from the 3rd day after the second treatment and beyond, the tumors in PBS and NC groups grew rapidly until the end of the observation period on the 15th day. Conversely, the tumors treated with $\mathrm{Lv}$-siIGF2R maintained a slower growth rate (Fig. 7A and B), and there was a significant difference in tumor volume between Lv-siIGF2R group and PBS and NC groups over the two-week observation period (Fig. 6, $\mathrm{P}<0.01)$. After recovery of two weeks, the tumor samples were removed for western blot analysis of the protein expression of IGF2R, PCNA, Bcl-2 and Cyclin D1. It was found that the protein expression levels of IGF2R, PCNA, Bcl-2 and Cyclin D1 were remarkably downregulated in Lv-siIGF2R group compared with those in PBS and NC groups (Fig. 7C).

\section{Discussion}

The interaction between IGF2R and IGF-II plays an important role in cellular physiology and pathological progression including tumor growth, invasion and metastasis (22). However, some studies show that the expression of IGF-II and IGF2R is strongly decreased in carcinomas and metastatic breast cancer, but increased in normal gland and adenomas (23). M6P/IGF2R reduces tumorigenicity and invasive potential of HCC, but knockdown of M6P/IGF2R enhances cell motility and invasiveness (24). To define the role of IGF-II/ IGF2R signaling in HAs, we examined the expression of IGF-II and IGF2R in human HA tissues by IHC, real-time PCR and western blot assays. It was found that the expression of IGF-II and IGF2R was significantly increased in proliferating phase HAs, but decreased in involuting phase HAs, suggesting IGF-II and IGF2R may be implicated in the development of human HA.

Survival factors including IGF2R play critical roles in regulating cell growth in normal and cancer cells. Genetic screen has identifies IGF2R as a cell surface marker in tumor cells, suggesting that IGF2R is required for tumor growth in a mouse model (25). However, few studies exist on the function of IGF-II/IGF2R signaling in HAs. In the present study, our findings indicated that knockdown of IGF2R gene significantly inhibited cell proliferation, and induced cell apoptosis and cycle arrest in HA cells in vitro and in vivo. Tovar et al (3) have previously found that IGF2R is activated and associated with the mTOR signaling in HCC, and a selective inhibition of IGF2R significantly decreases cell viability and proliferation, suggesting that IGF2R may be a promising target that promotes tumor progression in human HAs.

As non-histone nuclear proteins, PCNA and Ki-67 are associated with cell growth and plays a critical role in the initiation of cell proliferation. They have proved to be quite useful as the markers for proliferating cells (26). Apoptotic cell death is regulated by complex interactions between pro-survival members and two subgroups of pro-apoptotic members of $\mathrm{Bcl}-2$ protein family. Bax/Bcl-2 as a primary or secondary oncogenic event is critical in maintaining tumor development, and inducing therapeutic resistance (27). Cyclin D1 and E are cell cycle regulators that are also frequently altered in cancers, as they are found to be overexpressed and involved in cancers (28). Some studies have shown the association between these indexes and HAs. PCNA and Ki-67 are expressed by the majority of endothelial cells in the proliferating phase HAs, but their expression is negligible in the involuting phase (29-31). Bcl-2 contributes to the low apoptosis effect seen in HAs (32). However, the effects of IGF2R on the expression of PCNA, Ki-67, Bax, Bcl-2, Cyclin D1 and E have not been examined in HA cells. In the present study, we found that knockdown of IGF2R could significantly downregulate the expression of PCNA, Ki-67, Bcl-2, Cyclin D1 and E and upregulated the expression of Bax in HA cells and xenograft HA samples, suggesting that IGF2R may promote the development and progression of human HA via upregulation of PCNA, Ki-67, $\mathrm{Bcl}-2$, Cyclin D1 and E expression and downregulation of Bax expression. More importantly, PI3K/AKT has been found to play a role in the development of HAs $(20,21)$. Activation of the PI3K/AKT pathway promotes tumor growth and invasion through upregulation of the PCNA and Ki-67 expression (33), blocks cell apoptosis through decrease of the $\mathrm{Bax} / \mathrm{Bcl}-2$ ratio (34), and prompts cell cycle progression through upregulation of Cyclin D/E expression (35). We further found knockdown of IGF2R decreased the expression of p-PI3K and p-AKT in HA cells, suggesting that the PI3K/AKT pathway might mediate the regulation of IGF2R on the expression of PCNA, Ki-67, Bcl-2, Cyclin D1 and E implicated in HA proliferation and apoptosis.

In conclusion, our findings indicate that the expression of IGF-II and IGF2R is increased in proliferating phase HAs, and knockdown of IGF2R suppresses proliferation and induces apoptosis in HA cells in vitro and in vivo, suggesting that IGF2R may represent a novel therapeutic target for the treatment of human HAs.

\section{Acknowledgements}

This study was supported by Shanghai Science and Technology Committee scientific and technological innovation project (no. 12140901102), and Shanghai City Board of education research and innovation project (no. 12YZ042).

\section{References}

1. Mulliken JB, Fishman SJ and Burrows PE: Vascular anomalies. Curr Probl Surg 37: 517-584, 2000.

2. Xu L, Hausmann M, Dietmaier W, et al: Expression of growth factor receptors and targeting of EGFR in cholangiocarcinoma cell lines. BMC Cancer 10: 302, 2010. 
3. Tovar V, Alsinet C, Villanueva A, et al: IGF activation in a molecular subclass of hepatocellular carcinoma and pre-clinical efficacy of IGF-1R blockage. J Hepatol 52: 550-559, 2010.

4. Dong X, Javle M, Hess KR, et al: Insulin-like growth factor axis gene polymorphisms and clinical outcomes in pancreatic cancer. Gastroenterology 139: 464-473, 2010.

5. Dong X, Li Y, Tang H, et al: Insulin-like growth factor axis gene polymorphisms modify risk of pancreatic cancer. Cancer Epidemiol 36: 206-211, 2012.

6. Harris LK and Westwood M: Biology and significance of signalling pathways activated by IGF-II. Growth Factors 30: $1-12,2012$.

7. Hernandez L, Kozlov S, Piras G, et al: Paternal and maternal genomes confer opposite effects on proliferation, cell-cycle length, senescence, and tumor formation. Proc Natl Acad Sci USA 100: 13344-13349, 2003.

8. Kotsinas A, Evangelou K, Sideridou M, et al: The 3' UTR IGF2R-A2/B2 variant is associated with increased tumor growth and advanced stages in non-small cell lung cancer. Cancer Lett 259: 177-185, 2008.

9. Zavras AI, Pitiphat W, Wu T, et al: Insulin-like growth factor II receptor gene-167 genotype increases the risk of oral squamous cell carcinoma in humans. Cancer Res 63: 296-297, 2003.

10. Yoon AJ, Zavras AI, Chen MK, et al: Association between Gly1619ARG polymorphism of IGF2R domain 11 (rs629849) and advanced stage of oral cancer. Med Oncol 29: 682-685, 2012.

11. Hoyo C, Schildkraut JM, Murphy SK, et al: IGF2R polymorphisms and risk of esophageal and gastric adenocarcinomas. Int J Cancer 125: 2673-2678, 2009.

12. Lönn S, Rothman N, Shapiro WR, et al: Genetic variation in insulin-like growth factors and brain tumor risk. Neuro Oncol 10: 553-559, 2008

13. Kalla Singh S, Tan QW, Brito C, et al: Insulin-like growth factors I and II receptors in the breast cancer survival disparity among African-American women. Growth Horm IGF Res 20: 245-254, 2010.

14. Hu CK, McCall S, Madden J, et al: Loss of heterozygosity of M6P/IGF2R gene is an early event in the development of prostate cancer. Prostate Cancer Prostatic Dis 9: 62-67, 2006.

15. Chen Z, Ge Y, Landman N, et al: Decreased expression of the mannose 6-phosphate/insulin-like growth factor-II receptor promotes growth of human breast cancer cells. BMC Cancer 2: 18,2002 .

16. Oka Y, Waterland RA, Killian JK, et al: M6P/IGF2R tumor suppressor gene mutated in hepatocellular carcinomas in Japan. Hepatology 35: 1153-1163, 2002.

17. Yang EB, Qin LL, Zhao YN, et al: Genetic changes and expression of the mannose 6-phosphate/insulin-like growth factor II receptor gene in human hepatitis B virus-associated hepatocellular carcinoma. Int J Mol Med 11: 773-778, 2003.

18. Schiller HB, Szekeres A, Binder BR, et al: Mannose 6-phosphate/ insulin-like growth factor 2 receptor limits cell invasion by controlling alphaVbeta3 integrin expression and proteolytic processing of urokinase-type plasminogen activator receptor. Mol Biol Cell 20: 745-756, 2009.

19. Probst OC, Puxbaum V, Svoboda B, et al: The mannose 6-phosphate/insulin-like growth factor II receptor restricts the tumourigenicity and invasiveness of squamous cell carcinoma cells. Int J Cancer 124: 2559-2567, 2009.
20. Ji Y, Chen S, Li K, et al: Signaling pathways in the development of infantile hemangioma. J Hematol Oncol 7: 13, 2014.

21. Amin RM, Hiroshima K, Miyagi Y, et al: Role of the PI3K/Akt, mTOR, and STK11/LKB1 pathways in the tumorigenesis of sclerosing hemangioma of the lung. Pathol Int 58: 38-44, 2008.

22. Brown J, Jones EY and Forbes BE: Interactions of IGF-II with the IGF2R/cation-independent mannose-6-phosphate receptor mechanism and biological outcomes. Vitam Horm 80: 699-719, 2009.

23. Klopfleisch R, Hvid H, Klose $\mathrm{P}$, et al: Insulin receptor is expressed in normal canine mammary gland and benign adenomas but decreased in metastatic canine mammary carcinomas similar to human breast cancer. Vet Comp Oncol 8: 293-301, 2010.

24. Puxbaum V, Nimmerfall E, Bäuerl C, et al: M6P/IGF2R modulates the invasiveness of liver cells via its capacity to bind mannose 6-phosphate residues. J Hepatol 57: 337-343, 2012.

25. Gelman MS, Ye XK, Stull R, et al: Identification of cell surface and secreted proteins essential for tumor cell survival using a genetic suppressor element screen. Oncogene 23: 8158-8170, 2004.

26. Shin DM, Hittelman WN and Hong WK: Biomarkers in upper aerodigestive tract tumorigenesis: a review. Cancer Epidemiol Biomarkers Prev 3: 697-709, 1994.

27. Kelly PN and Strasser A: The role of Bcl-2 and its pro-survival relatives in tumourigenesis and cancer therapy. Cell Death Differ 18: 1414-1424, 2011.

28. Kim JK and Diehl JA: Nuclear cyclin D1: an oncogenic driver in human cancer. J Cell Physiol 220: 292-296, 2009.

29. Tan ST, Velickovic M, Ruger BM, et al: Cellular and extracellular markers of hemangioma. Plast Reconstr Surg 106: 529-538, 2000.

30. Murakami M, Sakai H, Kodama A, et al: Expression of the antiapoptotic factors Bcl-2 and survivin in canine vascular tumours. J Comp Pathol 139: 1-7, 2008.

31. Mohamed AM, Elwakil TF, Taher IM, et al: Cyclin D1 gene amplification in proliferating haemangioma. Cell Tissue Res 338: 107-115, 2009.

32. Nakamura T: Apoptosis and expression of Bax/Bcl-2 proteins in pyogenic granuloma: a comparative study with granulation tissue and capillary hemangioma. J Cutan Pathol 27: 400-405, 2000.

33. Fu Y, Zhang Q, Kang C, et al: Inhibitory effects of adenovirus mediated Akt1 and PIK3R1 shRNA on the growth of malignant tumor cells in vitro and in vivo. Cancer Biol Ther 8: 1002-1009, 2009.

34. Raja Singh P, Arunkumar R, Sivakamasundari V, et al: Antiproliferative and apoptosis inducing effect of nimbolide by altering molecules involved in apoptosis and IGF signalling via PI3K/AKT in prostate cancer (PC-3) cell line. Cell Biochem Funct 32: 217-228, 2014.

35. Chen C, Chang YC, Lan MS, et al: Leptin stimulates ovarian cancer cell growth and inhibits apoptosis by increasing cyclin D1 and Mcl-1 expression via the activation of the MEK/ERK1/2 and PI3K/Akt signaling pathways. Int J Oncol 42: 1113-1119, 2013. 